\title{
Intensity Thresholds and the Statistics of the Temporal Occurrence of Solar Flares
}

\author{
Marco Baiesi, ${ }^{1,2,3}$ Maya Paczuski, ${ }^{4,5}$ and Attilio L. Stella ${ }^{1,2}$ \\ ${ }^{1}$ INFM-Dipartimento di Fisica, Università di Padova, I-35131 Padova, Italy \\ ${ }^{2}$ Sezione INFN, Università di Padova, I-35131 Padova, Italy \\ ${ }^{3}$ Instituut voor Theoretische Fysica, K.U. Leuven, Belgium \\ ${ }^{4}$ John-von-Neumann Institute for Computing, Forschungszentrum Jülich, D-52425 Jülich, Germany \\ ${ }^{5}$ Perimeter Institute for Theoretical Physics, 31 Caroline Street North, Waterloo, Ontario N2L 2Y5, Canada
}

(Received 12 November 2004; published 6 February 2006)

\begin{abstract}
Introducing thresholds to analyze time series of emission from the Sun enables a new and simple definition of solar flare events and their interoccurrence times. Rescaling time by the rate of events, the waiting and quiet time distributions both conform to scaling functions that are independent of the intensity threshold over a wide range. The scaling functions are well-described by a two-parameter function, with parameters that depend on the phase of the solar cycle. For flares identified according to the current, standard definition, similar behavior is found.
\end{abstract}

DOI: $10.1103 /$ PhysRevLett.96.051103

PACS numbers: 96.60.qe, 05.45.Tp, 05.65.+b

The solar corona is a very high Reynolds number turbulent plasma producing intermittent bursts of radiation. Plasma forces twist the coronal magnetic fields until stresses are suddenly released, an avalanching process governed by magnetic reconnection [1]. The released magnetic energy induces radiative emission that can be detected as a flare. Flares exhibit scale invariant statistics. For instance, the probability distribution of flare energies is a power law spanning more than 8 orders of magnitude $[2,3]$, similar to the Gutenberg-Richter law for earthquakes. The distribution of magnetic concentration sizes on the photosphere is also scale invariant, and the coronal magnetic network embodies a scale-free network [4,5]. In fact, a model of self-organized criticality (SOC) with avalanches of reconnecting flux tubes reproduces the observed scalefree network structure $[4,6]$.

As part of the debate on the characterization of magnetohydrodynamic turbulence in this regime [1,4-9], interest has focused on comparing interoccurrence times between flares with those in models of SOC. Analyses of flare catalogs have indicated scale invariance of the waiting times, but the behavior was found to vary with the phase of the solar cycle [10] and with the methods used to analyze the catalogs. (See, e.g., Refs. $[10,11]$.) The prior belief that avalanches occur with Poissonian waiting times in the well-known Bak-Tang-Wiesenfeld (BTW) sandpile model [12] (giving an exponential distribution of waiting times) argued against the SOC hypothesis [8]. However, including a finite detection threshold leads to a power law distribution of quiet times even for the BTW model [13], when durations and quiet times are measured with the same clock. Since the turnover time scale for flux to be regenerated in the corona is of the order of 10 hours [14], while the correlated waiting time intervals between flares can extend up to years, the physical mechanism(s) responsible for these correlations resides in the turbulent convective region beneath the photosphere that generates magnetic flux and drives it into the corona. Systematic studies of the temporal pattern of flares can give insight into the dynamics of magnetic flux in the convective region, or the solar dynamo, which is difficult to observe directly.

Here we show that the interoccurrence times between flares has a hierarchical scaling structure when flares are defined as intervals during which the emission exceeds a given threshold. Rescaling time by the rate of these events, we find universal behavior for the interoccurrence times, which is independent of threshold. Both at solar maximum and at solar minimum, the scaling function can be fitted by a simple two-parameter function. This generalized Lorentzian arises naturally within a simplified model based on the time-dependent Poisson process. From this model, one can naturally infer an exponential distribution of flaring rates at solar maximum. At solar minimum, the distribution of quiet-or laminar-times is accurately described by on-off intermittency [15], a mechanism already proposed to describe the solar cycle [16].

In extremely intermittent time series, such as, e.g., earthquakes, events are spikes separated by a smooth background and are easily and uniquely defined. This is not the case for the solar data analyzed here, where the intensity decays slowly after a local peak, allowing overlaps with subsequent peaks. In this case, the introduction of a threshold is deeply connected to the definition of events, as indicated in Fig. 1.

The time series have been downloaded from the "Space Physics Interactive Data Resource" World Wide Web site [17], where each bin represents $x$-ray flux averaged over a given time unit. Among the available signals from various Geostationary Operational Environmental Satellites (GOES) [17], we consider the time series of the average soft $\mathrm{x}$-ray flux measured in $\mathrm{W} / \mathrm{m}^{2}$ with photons in the range from 1 to $8 \AA$. See Table I for details. We have also isolated two periods, roughly corresponding to the most recent minimum ("min") and maximum ("max") 


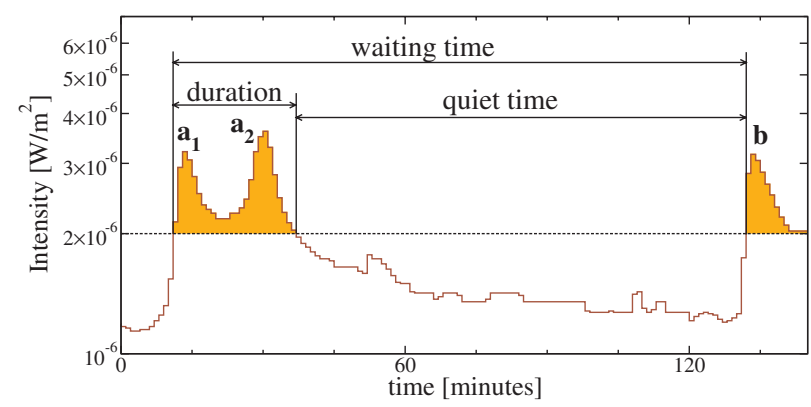

FIG. 1 (color online). Explanation of various times used in this work. Here the threshold intensity is $I=2 \times 10^{-6} \mathrm{~W} / \mathrm{m}^{2}$. According to the standard definition of flares, $\mathbf{a}_{1}$ and $\mathbf{a}_{2}$ would be two separate events. In our case, they are separated if, for instance, $I=3 \times 10^{-6} \mathrm{~W} / \mathrm{m}^{2}$. This shows that the set of events defined by different thresholds are not trivially related to each other or to the flares listed in the standard catalogs.

of the solar cycle. For comparison, we also analyze data in publicly available flare catalogs [18], which identify events with time intervals when the intensity is higher than a local average of the signal.

In contrast, we define an event, or flare, to be simply the interval during which the intensity exceeds a certain fixed threshold. To compensate for fixing the threshold, we study, in detail, the dependence on the threshold value and obtain results that are independent of the threshold over a wide range. Various times related to our definition of events (durations, waiting and quiet times) are explained in Fig. 1. One could expect differences when events defined by our simple procedure or by the standard flare catalogs are analyzed. However, we find that the statistical distributions are mostly similar. Hence, we expect similar results if other criteria are used to define interoccurence times, such as the time difference between subsequent maxima in the signal, referred to as the "laminar times" in Ref. [8].

TABLE I. Time series and selected intensity thresholds.

\begin{tabular}{lcccc}
\hline \hline & All $^{\mathrm{a}}$ & min $^{\mathrm{b}}$ & $\max ^{\mathrm{c}}$ & Sym $^{\mathrm{d}}$ \\
\hline Start & $1 / 1 / 1986$ & $1 / 9 / 1995$ & $1 / 1 / 2000$ & \\
End & $31 / 3 / 2004$ & $31 / 12 / 1996$ & $31 / 12 / 2003$ & \\
Bin width (minutes) & 5 & 1 & 1 & \\
Thresholds (W/m $\left.{ }^{2}\right)$ & $2 \times 10^{-6}$ & $3 \times 10^{-8}$ & $2 \times 10^{-6}$ & $\bigcirc$ \\
& $4 \times 10^{-6}$ & $10^{-7}$ & $4 \times 10^{-6}$ & $\square$ \\
& $10^{-5}$ & $3 \times 10^{-7}$ & $10^{-5}$ & $\diamond$ \\
& $3 \times 10^{-5}$ & $10^{-6}$ & $3 \times 10^{-5}$ & $\triangle$ \\
& $10^{-4}$ & $3 \times 10^{-6}$ & $10^{-4}$ & $\checkmark$ \\
\hline \hline
\end{tabular}

${ }^{\mathrm{a} B y}$ concatenating signals of satellites GOES 6, 7, 8, and 10 [17], we reconstruct the time series representing almost two solar cycles. Since some data are missing, values in the empty bins are set by the last recorded value before each of them. In this way, flat plateaus of intensity are created, introducing a minimal bias into the data.

${ }^{\mathrm{b}}$ Data from GOES 8, minimum of the solar cycle.

${ }^{\mathrm{c}}$ Data from GOES 10, maximum of the solar cycle.

${ }^{\mathrm{d}}$ Symbol used in the figures to denote the corresponding data.
Indeed, that definition also allows a systematic variation of intensity threshold used to select maxima that could be compared with the results shown here.

The number of events with intensity greater than or equal to a given threshold $I, N(\geq I)$, is shown in Fig. 2(a) for the entire data set, at min and at max. For the whole catalog, the number of flares $N(\geq I)$ behaves approximately as $I^{-\beta}$, with $\beta=1.2(1)$ for intensities greater than $\approx 10^{-6} \mathrm{~W} / \mathrm{m}^{2}$. Scaling breaks down below $I \approx 10^{-6}$, where $N(\geq I)$ increases with $I$. This clearly shows that $N(\geq I)$ is not the cumulative version of any probability distribution, because flares at different thresholds are different objects. In the two subregimes min and $\max$, we find power law behavior $N(\geq I) \sim I^{-\beta_{\max }}$, with $\beta_{\max }=1.2(1)$, for sufficiently large $I$. During the minimum of the cycle, however, another scaling regime appears. Indeed, $N_{\min }(\geq I) \sim I^{-\beta_{\min }}$, with $\beta_{\min }=0.7(1)$, for $I \lesssim 10^{-6} \mathrm{~W} / \mathrm{m}^{2}$. Within statistical error, the exponent $\beta_{\max }$ agrees with the (cumulative) distribution of peak fluxes measured by Aschwanden et al. [2], who obtained $\beta_{\max }+1=2.08 \pm 0.03$.

The thresholds and associated symbols used by us to define the events are given in Table I. For the entire data record, we choose five thresholds with $I>10^{-6}$, where $N(\geq I)$ is a decreasing function of $I$. The same thresholds are also used in the max regime. Since the flux at the maximum of solar activity is typically 2 orders of magnitude greater than at the minimum, a definition of flares by means of the same set of thresholds could be unfeasible. Five different thresholds are used at solar minimum to obtain reasonably good statistics.

We first discuss the distribution of duration times, $P\left(t_{d}\right)$. It has a power law tail, with critical exponent $\gamma_{\text {dur }}$, which extends to longer durations on lowering the threshold, as shown in Fig. 2(b). Previous measurements using a different definition of flares than that put forward here found $\gamma_{\text {dur }}=2.17$ to $\gamma_{\text {dur }}=2.54$, depending on the range of
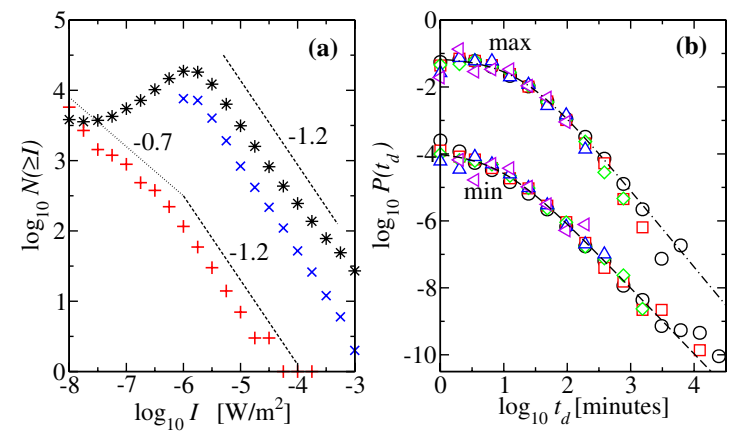

FIG. 2 (color online). (a) Number of events with intensity greater than a given threshold, for the entire record $(*)$, at the minimum $(+)$ and maximum $(\times)$ of the cycle. The straight lines represent power laws with the quoted exponent. (b) The distribution of flare durations, for different thresholds, at min and max of the solar cycle (the former shifted down by three units on the log-scale). The symbols are explained in Table I, while the curves are fits using Eq. (1). 
times fitted [19], while Litvinenko obtained $\gamma_{\mathrm{dur}}=2$ using dimensional analysis [20].

However, $P\left(t_{d}\right)$ crosses over from a power law at large times to a constant regime at short times. The entire distribution for all thresholds and all time periods is consistent with the function

$$
P\left(t_{d}\right) \sim\left(1+t_{d} / t_{d}^{*}\right)^{-\gamma_{\mathrm{dur}}},
$$

with $\gamma_{\mathrm{dur}}=2.0(1)$ [21] and $t_{d}^{*} \approx 10 \mathrm{~min}$ at solar minimum, while $\gamma_{\text {dur }}=2.3(4)$ and $t_{d}^{*} \approx 20$ min at solar maximum [23].

The waiting and quiet time distributions are shown in Fig. 3 for different thresholds and regimes. Each $P\left(t_{w}\right)$ is similar to its respective $P\left(t_{q}\right)$, especially at large times, where both decay as power laws $\sim t^{-\gamma}$. Hence, the scalefree duration of flares is not giving peculiarities in the passage from $t_{q}$ statistics to $t_{w}$ ones. The waiting/quiet time exponents $\gamma$ at lower thresholds have been evaluated in the three regimes. Within statistical error, these values, indicated in Fig. 3, agree with the ones determined by Wheatland and Litvinenko [10], who analyzed flare catalogs. However, one can observe that, by increasing the threshold, both $P\left(t_{w}\right)$ and $P\left(t_{q}\right)$ evolve continuously, becoming flatter up to longer times for higher thresholds. This aspect was not caught by any previous studies [10], whose results were obtained without systematically varying any threshold.

A scaling argument similar to one recently put forward by Bak et al. for waiting time statistics of earthquakes [24] can unify in a single scaling function the waiting/quiet time statistics. We argue that $N(\geq I)$ provides the right rescaling factor for the recurrence times, namely, the one that gives a collapse onto a single curve of all the distributions measured with different intensity thresholds $I$. In particular, we rescale the interoccurrence times by their average, which is inversely proportional to the average rate of

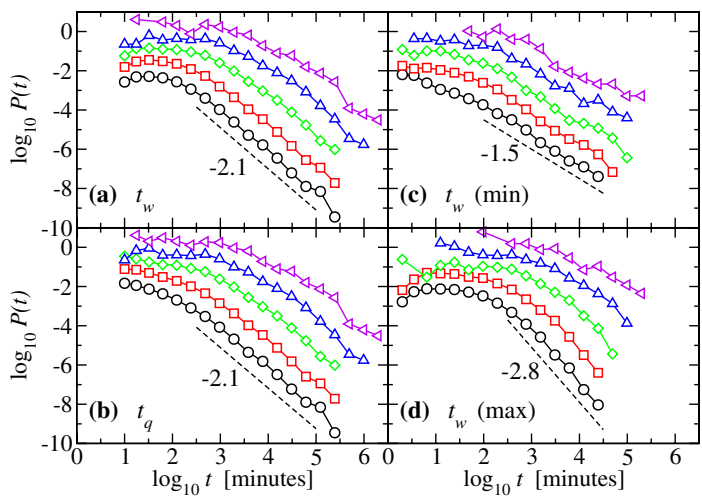

FIG. 3 (color online). The distributions of recurrence times, progressively shifted along the $y$ axes with the threshold, for clarity. (a), (c), and (d) Distributions of waiting times, for the entire record and at the minimum and maximum of the solar cycle, respectively. (b) Distribution of quiet times for the entire record. The power law tails are indicated with straight lines whose slope is $-\gamma$. The symbols are explained in Table I. events $R(I)=N(\geq I) / \Delta T$ [25], where $\Delta T$ is the time span of the record. Thus, the distribution of waiting times for a given threshold is given by

$$
P\left(t_{w}, I\right) \sim R(I) g\left(t_{w} R(I)\right)
$$

Unlike the universal waiting time distributions for earthquakes [24,25], but similar to $P\left(t_{d}\right)$, the scaling function $g$ for the flare waiting and quiet times is also well-described by the function $g(x) \sim\left(1+x / x^{*}\right)^{-\gamma}$, as shown in Fig. 4. Furthermore, the rescaled distributions have parameters that depend on the phase of the solar cycle: For the whole catalog, $\gamma \simeq 2.16(5)$ and $x^{*} \approx 0.26$. At the minimum of the cycle $\gamma=1.51(5)\left(x^{*} \approx 0.02\right)$, while at the maximum $\gamma=$ 2.83(10) $\left(x^{*} \approx 0.85\right)$.

To compare with the standard definition of flares, we repeat the above analysis using the GOES flare catalog from 1975-2003 [18]. Now the threshold $I$ represents the peak intensity associated with the flare. The rate of events with threshold $I, R(I)$, is the number of events in the catalog with peak intensity greater than $I$ divided by the total duration of the catalog. Using the same thresholds as before for the whole catalog and rescaling the distributions using Eq. (2), we obtain the results shown in Fig. 4 (upper group of data). In this case, power law behavior with an exponent $\gamma=2.19(5)\left(x^{*} \approx 0.28\right)$ is observed at late times, although at short times there are deviations from data collapse at lower thresholds. The deviations may be due to undercounting short waiting times following a large flare, an obscuration effect previously pointed out by Wheatland [26]. All of the data sets can also be fitted with Lévy functions, which turn out to give comparable results except for the waiting times at solar maximum, where the fit with Lévy distributions is inferior [27]. We choose here to focus on the fit using a single function

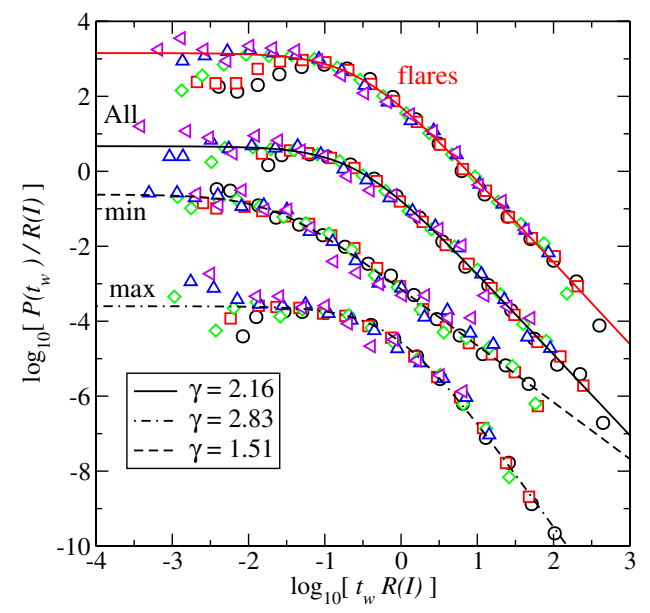

FIG. 4 (color online). The rescaled distributions of waiting times, arbitrarily shifted vertically to be distinguished. From below, for the maximum of the solar cycle, for the minimum, for the entire catalog, and for the GOES flare catalog. Fits as described in the text are shown for the different phases. 
[Eq. (1)] since it appears to describe all the data sets equally well.

Wheatland $[7,10,26]$ has modeled the solar flare waiting time distribution in terms of a time-dependent Poisson process with a flaring rate $\lambda(t)$. When the flaring rate varies slowly over a waiting time $t_{w}$, the distribution of waiting times can be written as

$$
P\left(t_{w}, I\right)=\frac{1}{\bar{\lambda}_{I}} \int_{0}^{\infty} F_{I}(\lambda) \exp \left(-\lambda t_{w}\right) \lambda^{2} d \lambda,
$$

where the average flaring rate $\bar{\lambda}_{I}=\int_{0}^{\infty} F_{I}(\lambda) \lambda d \lambda$, and $F_{I}(\lambda) \Delta \lambda$ is the fraction of the time the rate to produce flares exceeding intensity $I$ is within $\Delta \lambda$ of $\lambda$. The function we find to fit the data has $f_{I}(\lambda)=\lambda^{2} F_{I}(\lambda) / \bar{\lambda}$ corresponding to the Gamma distribution:

$$
f_{I}(\lambda) \sim\left(\frac{\lambda x^{*}}{R(I)}\right)^{\gamma-1} \exp \left(-\frac{\lambda x^{*}}{R(I)}\right) .
$$

A mathematical equivalence with the superstatistics formulas of Beck and Cohen [22] can be made by mapping $\lambda \rightarrow \beta, t_{w} \rightarrow E$, and $f(\lambda) \rightarrow f(\beta)$. No necessary physical connection is implied in this equivalence, since one could just as well think of a subordination mechanism [28] as being at the basis of Eq. (3). It is also worth remarking that several turbulent systems have been analyzed recently using the superstatistics framework. These include velocity differences in Taylor-Couette flow [29], and intermittency of the wind [30] or solar wind [31].

At solar maximum, the critical exponent $\gamma$ for the waiting time distribution is close to $\gamma=3$. This implies that distribution of flaring rates $F_{I}(\lambda)$ is close to exponential for a range of $I$. Since high intensity flares predominately arise from active regions during solar maximum, the origin of this distribution could be investigated by tracking the flaring rates of individual active regions.

At solar minimum, the critical exponent $\gamma \approx 3 / 2$ for a range of intensities $I$, implying that the distribution of flare rates

$$
F_{I}^{\min }(\lambda) \sim \lambda^{-3 / 2} \exp \left(-\frac{\lambda x^{*}}{R(I)}\right) .
$$

This formula describes the probability distribution for the number of offspring in a subcritical branching process [32].

Alternatively, the marginal behavior in on-off intermittency also gives a distribution of laminar times with an exponent $\gamma=3 / 2$ [15], in very good agreement with the quiet time distribution at solar minimum. In fact, an intermittent on-off dynamo [16] has been used to describe the solar cycle and long term records of solar activity such as grand minima. Our results lead us to speculate that such a dynamo operating in a marginal state may also be able to capture the quiet times of flares - excluding active regions. Self-organized criticality may provide a mechanism for this dynamo to sustain itself in a marginal state. Active regions, superimposed on this fluctuating state, may rep- resent plasma instabilities in the on-off dynamo, with their own emergent behavior.

[1] E. Lu and R. Hamilton, Astrophys. J. 380, L89 (1991).

[2] M. J. Aschwanden et al., Astrophys. J. 535, 1047 (2000).

[3] B. R. Dennis, Sol. Phys. 100, 465 (1985).

[4] D. Hughes and M. Paczuski, astro-ph/0309230.

[5] M. Paczuski and D. Hughes, Physica (Amsterdam) 342A, 158 (2004).

[6] D. Hughes et al., Phys. Rev. Lett. 90, 131101 (2003).

[7] M. S. Wheatland, Astrophys. J. 536, L109 (2000).

[8] G. Boffetta et al., Phys. Rev. Lett. 83, 4662 (1999).

[9] R. Sánchez, D. E. Newman, and B. A. Carreras, Phys. Rev. Lett. 88, 068302 (2002); P. Charbonneau et al., Sol. Phys. 203, 321 (2001); M. P. Freeman, N. W. Watkins, and D. J. Riley, Phys. Rev. E 62, 8794 (2000); M. De Menech and A.L. Stella, Physica (Amsterdam) 309A, 289 (2002); A. Bershadskii and K. R. Sreenivasan, Eur. Phys. J. B 35, 513 (2003).

[10] M.S. Wheatland and Y. E. Litvinenko, Sol. Phys. 211, 255 (2002).

[11] P. Grigolini, D. Leddon, and N. Scafetta, Phys. Rev. E 65, 046203 (2002).

[12] P. Bak, C. Tang, and K. Wiesenfeld, Phys. Rev. Lett. 59, 381 (1987).

[13] M. Paczuski, S. Boettcher, and M. Baiesi, Phys. Rev. Lett. 95, 181102 (2005).

[14] H. J. Hagenaar, C. J. Schrijver, and A. M. Title, Astrophys. J. 584, 1107 (2003).

[15] J. F. Heagy, N. Platt, and S. M. Hammel, Phys. Rev. E 49, 1140 (1994).

[16] D. Schmitt, M. Schüssler, and A. Ferriz-Mas, Astron. Astrophys. 311, L1 (1996).

[17] URL http://spidr.ngdc.noaa.gov/spidr/

[18] URL ftp://ftp.ngdc.noaa.gov/STP/SOLAR_DATA/ SOLAR_FLARES/XRAY_FLARES

[19] N. B. Crosby, M. J. Aschwanden, and B. R. Dennis, Sol. Phys. 143, 275 (1993).

[20] Y.E. Litvinenko, Sol. Phys. 180, 393 (1998).

[21] Our $\gamma_{\text {dur }}$ corresponds to $1 /(q-1)$ of C. Tsallis, J. Stat. Phys. 52, 479 (1988) and of Ref. [22].

[22] C. Beck and E. G. D. Cohen, Physica (Amsterdam) 322A, 267 (2003).

[23] With the software XMGRACE, we fitted data in log-scale with the function $y(z)=A-\gamma \log _{10}\left(1+10^{z} / x^{*}\right)$.

[24] P. Bak et al., Phys. Rev. Lett. 88, 178501 (2002).

[25] A. Corral, Phys. Rev. E 68, 035102(R) (2003).

[26] M. S. Wheatland, Sol. Phys. 203, 87 (2001).

[27] H.-J. Seybold (private comunication).

[28] W. Feller, An Introduction to Probability Theory and Its Applications (Wiley, New York, 1971).

[29] C. Beck, G. S. Lewis, and H. L. Swinney, Phys. Rev. E 63, 035303(R) (2001).

[30] S. Rizzo and A. Rapisarda, in Experimental Chaos: 8th Experimental Chaos Conference, edited by S. Boccaletti et al., AIP Conf. Proc. No. 742 (AIP, New York, 2004), p. 176.

[31] M. P. Leubner and Z. Voros, Astrophys. J. 618, 547 (2005).

[32] T. Harris, The Theory of Branching Processes (Springer, Berlin, 1997). 